(C) 2010 IEEE. Personal use of this material is permitted. Permission from IEEE must be obtained for all other uses, in any current or future media, including reprinting/republishing this material for advertising or promotional purposes, creating new collective works, for resale or redistribution to servers or lists, or reuse of any copyrighted component of this work in other works. 


\title{
Validation of PeneloPET Positron Range Estimations
}

\author{
J. Cal-González, Member, IEEE, J.L. Herraiz, Member, IEEE, S. España, Member, IEEE, M. Desco, J.J. Vaquero, \\ Senior Member, IEEE and J.M. Udías, Member, IEEE
}

\begin{abstract}
Positron range depends on the materials in which positron propagates and on positron emitter isotope. As positron range limits the spatial resolution of PET images, good quantitative estimates of it should be included in any realistic simulation of PET acquisitions. In this work we compare positron range estimates obtained with PeneloPET to previous available simulations and experimental data. PeneloPET was used to simulate the positron range of ${ }^{18} \mathrm{~F},{ }^{11} \mathrm{C},{ }^{13} \mathrm{~N},{ }^{15} \mathrm{O},{ }^{68} \mathrm{Ga}$ and ${ }^{82} \mathrm{Rb}$ in the following tissues: cortical bone, soft bone, skin, muscle, brain, water, adipose tissue and lung. The 3D and 1D annihilation Point Spread Functions (aPSF) were calculated for each isotope-material combination. We have studied with more detail the 3D aPSF (radial distributions) and the cumulative fraction of annihilation events. These aPSF distributions were also studied for non-uniform media. Results obtained were consistent with other results previously reported in the literature as well as with experimentally measured data.
\end{abstract}

\section{INTRODUCTION}

$\mathrm{P}^{\mathrm{s}}$ OSITRON RANGE limits the spatial resolution of PET images and has a different effect for different isotopes and positron propagation materials. Early experimental efforts to measure the distribution of annihilation points for medically important positron emitters in water were of limited accuracy since the detector resolution were comparable to the positron range effect of interest [1]. The difficulty to the experimental measurement of positron range led to the use of Monte Carlo simulations to estimate positron range [2] - [5]. These studies share the limitation of having only used water as a reference medium for positron interaction. Therefore, these simulations results in clinical practice can be rather misleading since positron interaction with matter depends on tissue density and atomic composition of the positron propagation material. Thus, it is expected that the blurring introduces by positron range will vary in size and shape depending of the kind of

Manuscript received November 12, 2010. This work has been supported by MEC (FPA2007-62216), UCM (Grupos UCM, 910059), CPAN (ConsoliderIngenio 2010) CSPD-2007-00042, European Regional Development and ENTEPRASE grant, PSE-300000-2009-5, and the CENIT program project AMIT, Ministerio de Ciencia e Innovación, Spanish Government. Part of the calculations of this work were performed in the "Clúster de Cálculo de Alta Capacidad para Técnicas Físicas" funded in part by UCM and in part by UE under FEDER programme".

J. Cal-González, J.L. Herraiz and J.M. Udías are with the Grupo de Física Nuclear, Dpto. Física Atómica, Molecular y Nuclear, UCM, Madrid, Spain (telephone: +34 91394 4484, e-mail: jacobo@nuclear.fis.ucm.es)

S. España was with the Grupo de Física Nuclear, Universidad Complutense de Madrid, Spain. He is now with the Department of Radiation Oncology, Massachusetts General Hospital and Harvard Medical School, Boston, MA, USA (e-mail: sespana-palomares@partners.org).

M. Desco is with the Unidad de Medicina y Cirugía Experimental, Hospital General Universitario Gregorio Marañón, Madrid, Spain (e-mail: desco@mce.hggm.es).

J.J. Vaquero is with the Departamento de Bioingeniería e Ingeniería Aeroespacial, Universidad Carlos III de Madrid, Spain (e-mail: juanjose.vaquero@uc3m.es). tissue in which the positron is propagated [6], [7]. The goal of this work has been to assess the positron range estimates for the most common isotopes in various human tissues using PeneloPET [8].

\section{METHODS}

\section{A. Positron range modeling}

For a given radioactive point source emitting positrons in random directions, the 3D Cartesian coordinates $(\mathrm{x}, \mathrm{y}, \mathrm{z})$ can be registered for each annihilation event and then provide $3 \mathrm{D}$ annihilation Point Spread Function (aPSF). A theoretical model for this 3D distribution was proposed by Palmer and Brownell [9]. In their work, for a point emitter of monoenergetic positrons (with energy $\mathrm{E}_{0}<4 \mathrm{MeV}$ ) in an isotropic media, the aPSF can be represented by a threedimensional Gaussian distribution [9].

Following the theoretical model proposed by Palmer and Brownell [9], the aPSF for a point source beta emitter in an isotropic medium can be characterized by the following expression [9]:

$$
\operatorname{aPSF}(r) \equiv \int_{0}^{E_{\max }}\left(\sqrt{2 \pi} \sigma^{2}\right)^{-3} e^{-\frac{r^{2}}{2 \sigma^{2}}} N\left(E_{0}\right) d E_{0}
$$

Where the energy spectrum of the beta source is denoted by $\mathrm{N}\left(\mathrm{E}_{0}\right), \mathrm{r}$ is the radial distance from the origin and $\sigma$ is the standard deviation for a given energy $\mathrm{E}_{0}$.

The following radial histograms can be also obtained for each isotope - material combination:

$$
\begin{gathered}
g_{3 D}(r) \equiv 4 \pi \int_{r}^{r+d r} r^{\prime 2} a P S F\left(r^{\prime}\right) d r^{\prime} \\
G_{3 D}(r) \equiv \int_{0}^{r} g\left(r^{\prime}\right) d r^{\prime}
\end{gathered}
$$

The function (2) represents the radial histogram weighted by the total number of counts given for a certain radial distance.

The 3D cumulative distribution $\mathrm{G}_{3 \mathrm{D}}(\mathrm{r})$ (equation 3 ) can be also obtained for the aPSF (r).

With the projection of the aPSF(r) onto a plane XY we can obtain the $2 \mathrm{D}$ distributions $\mathrm{g}_{2 \mathrm{D}}(\mathrm{r})$, which is the radial histogram weighted by the number of counts given for a certain radial distance in the projection onto the plane $\mathrm{XY}$, and $\mathrm{G}_{2 \mathrm{D}}(\mathrm{r})$, which is the cumulative distribution in $2 \mathrm{D}$.

Other authors compute the following two 1D distributions for this aPSF. The first one is the projection onto one dimension $\left(\mathrm{aPSF}_{\sin }\right)$, for example the $\mathrm{x}$ direction, which is the distribution that contributes directly to the sinogram [10]. 


$$
a P S F_{\sin }(x) \equiv \int_{-\infty}^{\infty} d z \int_{-\infty}^{\infty} a P S F(x, y, z) d y
$$

These distributions are given in the simulations of Levin \& Hoffmann [2], Champion \& Le Loirec [5] and Blanco [10].

The second one is the 1D profile, at the maximum, of the projection onto the xy plane $\left(\mathrm{aPSF}_{\mathrm{img}}\right)$ [10].

$$
\operatorname{aPSF}_{\text {img }}(x) \equiv \int_{Y_{m}-\Delta y}^{Y_{m}+\Delta y} d y \int_{-\infty}^{\infty} a P S F(x, y, z) d z
$$

This distribution contributes directly to the image spatial resolution [10], and is calculated in the experimental work of Derenzo [1] and in the simulations performed by Blanco [10].

The above mentioned 1D distribution could be characterized by a fit to the sum of two exponential functions [1]:

$$
P(x)=C \cdot e^{-k_{1} x}+(1-C) e^{-k_{2} x} ; \mathrm{x} \geq 0
$$

Where $C, \mathrm{k}_{1}$ and $\mathrm{k}_{2}$ are fitting parameters. Of these fitting parameters, $\mathrm{k}_{1}$ is strongly dependent of the bin size, and then does not have a relevant physical significance. On the other hand, $\mathrm{k}_{2}$, that represents the broad exponential constant it is rather independent of bin size and thus it is a good parameter to compare results from other authors.

\section{B. PeneloPET simulation for a homogeneous medium}

PeneloPET was used to simulate the positron range of ${ }^{18} \mathrm{~F}$, ${ }^{11} \mathrm{C},{ }^{13} \mathrm{~N},{ }^{15} \mathrm{O},{ }^{68} \mathrm{Ga}$ and ${ }^{82} \mathrm{Rb}$ in the following tissues: cortical bone (1.85 g.cm-3), soft bone (1.45 g.cm-3), skin (1.10 g.cm3 ), muscle striated (1.04 g.cm-3), brain (1.03 g.cm-3), water (1.00 g.cm-3), adipose tissue (0.92 g.cm-3) and lung (0.30 g.cm-3).

For each combination isotope - material investigated, a large number of $10^{6}$ disintegrations were generated. The aPSF(r), $\mathrm{aPSF}_{\text {sin }}$ and the $\mathrm{aPSF}_{\text {img }}$ were obtained, and these histograms were fitted to the sum of two exponential functions show in equation (6).

The $g_{3 D}(r), G_{3 D}(r), g_{2 D}(r)$ and $G_{2 D}(r)$ were also calculated with PeneloPET simulations for each isotope - material combination.

In order to compare our results with the ones obtained by other authors, it is of interest to compute the fraction of annihilations within a sphere or radius $r$ (3D cumulative distribution) or in the projection inside a circle of radius $r$ (2D cumulative distribution).

\section{PeneloPET simulation for non-uniform media}

Two different simulations with non-uniform media were performed in PeneloPET. The first one consists in a ${ }^{68} \mathrm{Ga}$ point source placed in the centre of a $2 \mathrm{~mm}$ bone tissue sphere, with a concentric $5 \mathrm{~cm}$ water sphere. In the second simulation, the ${ }^{68} \mathrm{Ga}$ point source was placed in the centre of a $5 \mathrm{~mm}$ lung tissue sphere, with a concentric $5 \mathrm{~cm}$ water sphere.

\section{RESULTS}

\section{A. 1D Distribution: $a P S F_{\text {sin }}$}

Table I shows the $\mathrm{k}_{2}$ values obtained with the PeneloPET simulation for different isotope - material combinations.

Figure 1 (top) shows the $\mathrm{k}_{2}$ values obtained with the fitting to the sum of two exponential functions, for the $\mathrm{aPSF}_{\text {sin }}$ distribution, as a function of the $\mathrm{E}_{\max }$ energy for the different isotopes simulated with PeneloPET; the comparison with other values found in the literature is also shown. These $\mathrm{k}_{2}$ values were also obtained as a function of the density of the materials simulated (see figure 1, bottom).

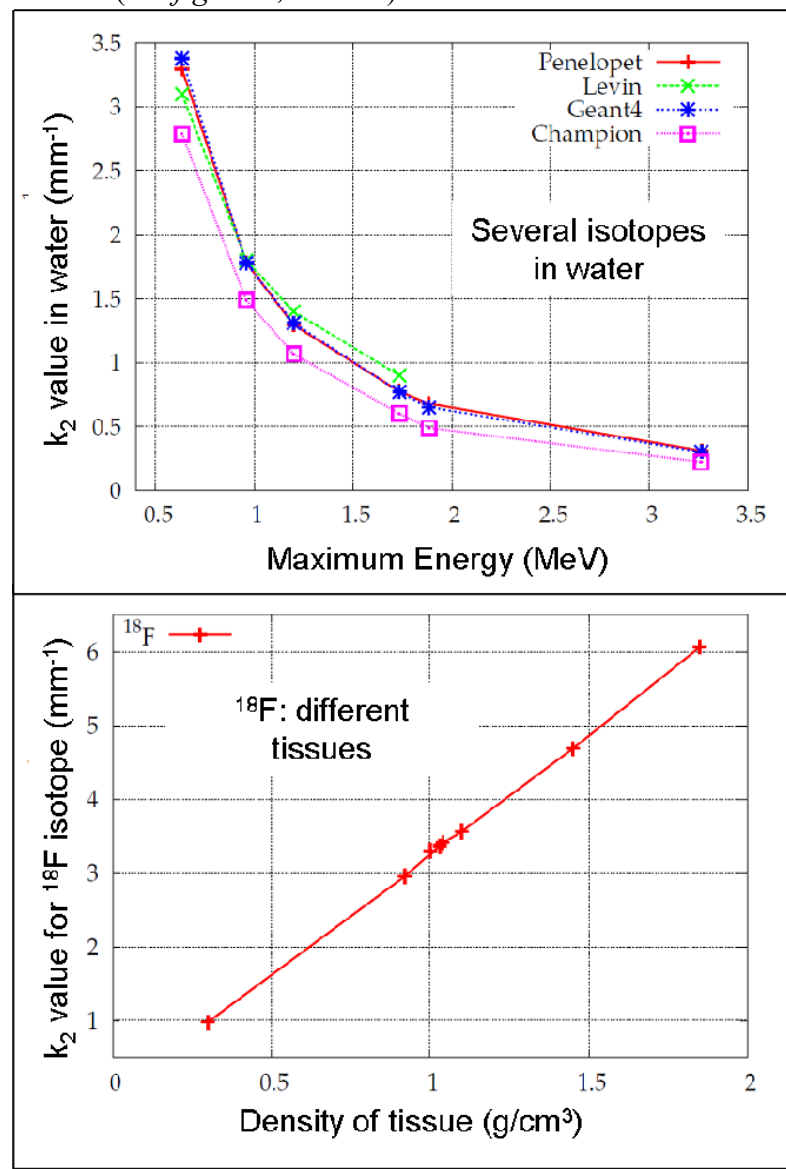

Fig. 1. $\mathrm{k}_{2}$ fitting values for the $\mathrm{aPSF}_{\text {sin }}$ distribution. Upper panel: Dependence of $k_{2}$ values with the maximum energy of the different isotopes simulated. The results obtained were compared with Levin \& Hoffmann [2], Geant4 [10] and Champion [5] simulations. Champion's results deviates from the other simulations because these are the only ones that consider positronium formation. Lower panel: $\mathrm{k}_{2}$ dependence with the density of the simulated materials obtained with PeneloPET.

\section{B. $2 D$ and $3 D$ cumulative distributions}

Figure 2 shows the fraction of annihilation events within a sphere of radius $r$ for different isotopes (left) and materials (right). 
TABLE I. K2 2 FITTING PARAMETERS OBTAINED FOR THE FIT TO THE TWO EXPONENTIAL FUNCTIONS PROPOSED BY DERENZO [1].

\begin{tabular}{|c|c|c|c|c|}
\hline & Cortical bone & Soft bone & Water & Lung \\
\hline Isotope & $\begin{array}{c}\mathrm{k}_{2} \\
\left(\mathrm{~mm}^{-1}\right)\end{array}$ & $\begin{array}{c}\mathrm{k}_{2} \\
\left(\mathrm{~mm}^{-1}\right)\end{array}$ & $\begin{array}{c}\mathrm{k}_{2} \\
\left(\mathrm{~mm}^{-1}\right)\end{array}$ & $\begin{array}{c}\mathrm{k}_{2} \\
\left(\mathrm{~mm}^{-1}\right)\end{array}$ \\
\hline${ }^{18} \mathrm{~F}$ & 6.06 & 4.69 & 3.27 & 0.98 \\
\hline${ }^{11} \mathrm{C}$ & 3.29 & 2.54 & 1.79 & 0.53 \\
\hline${ }^{13} \mathrm{~N}$ & 2.42 & 1.83 & 1.30 & 0.39 \\
\hline${ }^{15} \mathrm{O}$ & 1.43 & 1.10 & 0.77 & 0.23 \\
\hline${ }^{68} \mathrm{Ga}$ & 1.24 & 0.95 & 0.67 & 0.20 \\
\hline${ }^{82} \mathrm{Rb}$ & 0.59 & 0.45 & 0.31 & 0.10 \\
\hline
\end{tabular}

Table II presents the comparison of our 3D cumulative distributions with those obtained by Champion \& Le Loirec [5]. In table III the comparison of our 2D cumulative distributions with Derenzo [1] experimental results is shown.
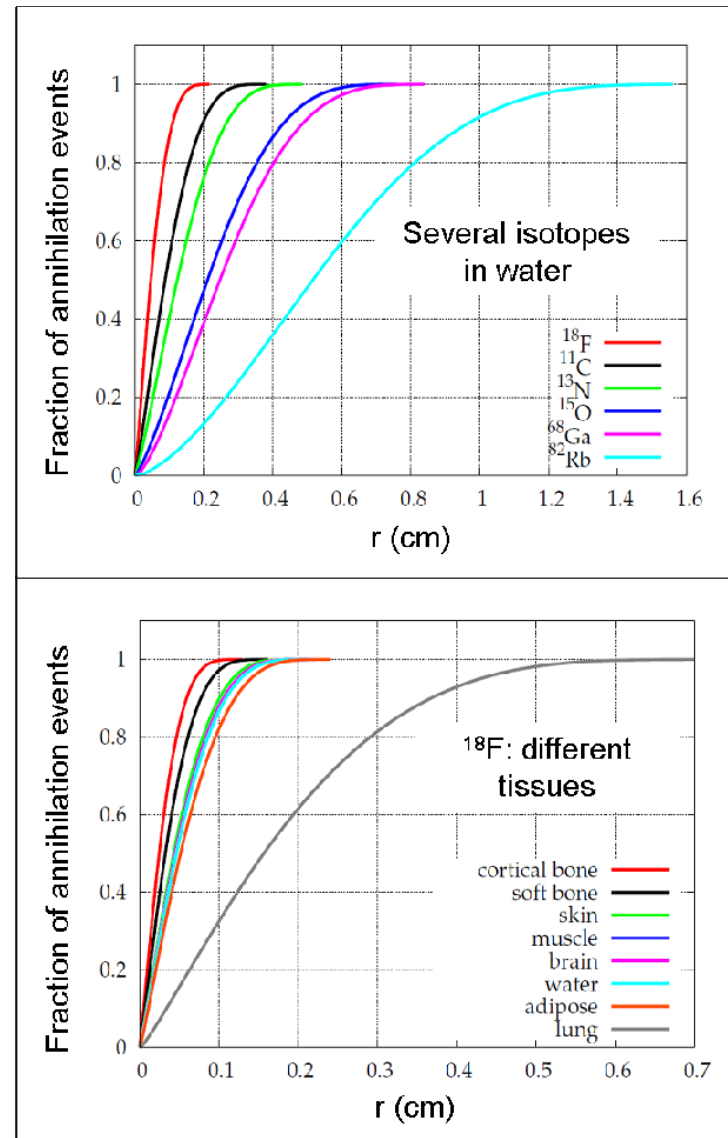

Fig. 2. Upper panel: 3D cumulative distributions for different isotopes in water. Lower panel: $3 \mathrm{D}$ cumulative distributions for ${ }^{18} \mathrm{~F}$ in different materials.
TABLE II. 3D CUMULATIVE DISTRIBUTIONS CALCULATED WITH PENELOPET AND CHAMPION [5] SIMULATIONS, WITH AND WITHOUT POSITRONIUM (PS) FORMATION, FOR ${ }^{15} \mathrm{O}$ IN WATER.

\begin{tabular}{|c|c|c|}
\cline { 2 - 3 } \multicolumn{1}{c|}{} & \multicolumn{2}{c|}{ Fraction of annihilation events: ${ }^{\mathbf{1 5}}$ O (Water) } \\
\cline { 2 - 3 } \multicolumn{1}{c|}{} & PeneloPET & $\begin{array}{c}\text { Champion: } \\
\text { with Ps / without Ps }\end{array}$ \\
\hline $\mathbf{1} \mathbf{~} \mathbf{m}$ & 0.21 & $0.16 / 0.22$ \\
\hline $\mathbf{2} \mathbf{~} \mathbf{~ m}$ & 0.47 & $0.33 / 0.45$ \\
\hline $\mathbf{3 m}$ & 0.70 & $0.52 / 0.66$ \\
\hline $\mathbf{5} \mathbf{~} \mathbf{m}$ & 0.96 & $0.81 / 0.93$ \\
\hline $\mathbf{8} \mathbf{~ m m}$ & 1.00 & $0.99 / 1.00$ \\
\hline
\end{tabular}

TABLE III. COMPARISON BETWEEN THE SIMULATED 2D CUMULATIVE DISTRIBUTIONS AND DERENZO [1] EXPERIMENTAL RESULTS.

\begin{tabular}{|c|c|c|}
\cline { 2 - 3 } \multicolumn{1}{c|}{} & \multicolumn{2}{c|}{ Fraction of annihilation events $\left({ }^{\mathbf{1 1}} \mathbf{C} /{ }^{\mathbf{6 8}} \mathbf{G a} /{ }^{\mathbf{8 2}} \mathbf{R b}\right)$} \\
\cline { 2 - 3 } \multicolumn{1}{c|}{} & Penelopet & Derenzo \\
\hline $\mathbf{1} \mathbf{~ m m}$ & $0.69 / 0.27 / 0.10$ & $0.76 / 0.29 / 0.12$ \\
\hline $\mathbf{2} \mathbf{~ m m}$ & $0.96 / 0.55 / 0.25$ & $0.95 / 0.59 / 0.23$ \\
\hline $\mathbf{3} \mathbf{~ m m}$ & $1.00 / 0.76 / 0.40$ & $0.99 / 0.80 / 0.36$ \\
\hline $\mathbf{5} \mathbf{~ m m}$ & $1.00 / 0.96 / 0.66$ & $1.00 / 0.98 / 0.59$ \\
\hline $\mathbf{8} \mathbf{~ m m}$ & $1.00 / 1.00 / 0.90$ & $1.00 / 1.00 / 0.83$ \\
\hline
\end{tabular}

\section{Results for the simulation of non-uniform media}

Figure 3 shows $\mathrm{g}(\mathrm{r})$ and the accumulated distributions calculated for the two non-uniform media PeneloPET simulations.

\section{CONCLUSIONS AND FUTURE WORK}

Predictions of PeneloPET for positron range are compared to different approaches given in the literature for several isotope-material combinations.

Results were consistent with other simulations, except for the case of Champion's when positronium formation is considered, in which case peneloPET seems to under predict range effects. However, the comparison to experimental data of Derenzo [1] shows that peneloPET agrees with experiment within $10 \%$. The positron range distributions were also studied for non-uniform media.

Further comparison to experimental data will be performed, as well as a more detailed study of positronium influence in positron range. 


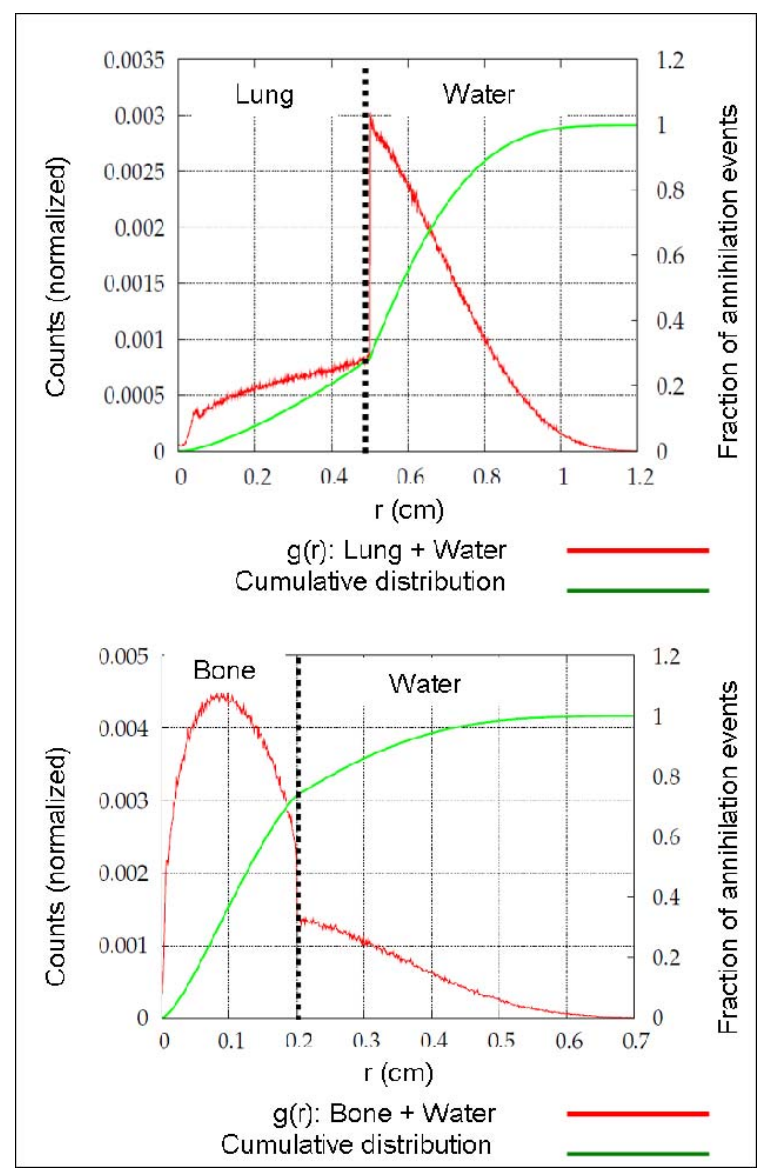

Fig. 3. Upper panel: $g_{3 D}(r)$ and 3D cumulative distributions for the lung / water simulation (boundary: $\mathrm{r}=0.5 \mathrm{~cm}$ ). Lower panel: $\mathrm{g}_{3 \mathrm{D}}(\mathrm{r})$ and cumulative distributions for the bone / water simulation (boundary: $\mathrm{r}=0.2 \mathrm{~cm}$ ).

\section{REFERENCES}

[1] S.E. Derenzo, Precision Measurement of Annihilation Point Spread Distributions for Medically Important Positron Emitters, Positron Annihilation R.R Hasiguti and K. Fujiwara, Eds. Sendai, Japan: The Japan Institute of Metals, 819-23, 1979.

[2] C.S. Levin and E.J. Hoffman, Calculation of Positron Range and Its Effect on the Fundamental Limit of Positron Emision Tomography System Spatial Resolution, Phys. Med. Biol., 44:781-99, 1999.

[3] R.L. Harrison, M.S. Kaplan, S.D. Vannoy, T.K. Lewellen, Positron range and coincidence non - collinearity in SimSET, IEEE NSS MIC Conf. Rec, 1265-1268, 1999.

[4] M.R. Palmer, X. Zhu and J.A. Parker, Modelling and Simulation of Positron Range Effects for High Resolution PET Imaging, IEEE TNS, vol 52, n 5, 1391-1395, 2005.

[5] C. Champion and C. L. Loirec, Positron follow-up in liquid water: II. spatial and energetic study for the most important radioisotopes used in PET. Phys. Med. Bio., vol. 52, no. 22, 6605-6625, 2007.

[6] A. Sanchez-Crespo, P. Andreo and S.A. Laarson, Positron flight in human tissues and its influence on PET image spatial resoution, Eur. J. Nucl. Med. Mol. Imaging, 31, 44-51, 2004.

[7] A. Alessio and L. MacDonald, Spatially Variant Positron Range Modeling Derived from CT for PET Image Reconstruction. IEEE Nucl. Sci. Symposium Conference Record, 2912-2915, 2009.

[8] S. España, J L. Herraiz, E. Vicente, J J. Vaquero, M. Desco, J.M. Udias, PeneloPET, a Monte Carlo PET simulation tool based on PENELOPE: features and validation, Phy Med Biol, 54, 1723-42, 2009.

[9] M.R. Palmer and G.L. Brownell, Annihilation density distribution calculations for medically important positron emitters, IEEE Trans. Med. Imaging, 11, 373-8, 1992.

[10] A. Blanco, Positron Range Effects on the Spatial Resolution of RPCPET, IEEE NSS MIC Conf. Rec, 2570-2573, 2006. 